\title{
Estudio de las actitudes de estudiantes de Ciencias Sociales y Psicología: relevancia de la información y contacto con personas discapacitadas"
}

\author{
Study of the Attitudes of University Students Coming \\ from Social Sciences and Psychology: Relevance of the \\ Information and Contact with the Disabled
}

Recibido: julio 13 de 2009 | Revisado: marzo 15 de 2010 | Aceptado: agosto 2 de 2010

\author{
M Tamara Polo SÁNCHez ${ }^{\text {*** }}$ \\ CAROLINA FERNÁNDEZ JIMÉNEZ \\ Universidad de Granada, España \\ CARMEN DÍAZ BATANERO *** \\ Universidad de Huelva, España
}

Para citar este artículo. Polo, M. T., Fernández, C., \& Díaz, C. (2011). Estudio de las actitudes de estudiantes de Ciencias Sociales y Psicología: relevancia de la información y contacto con personas discapacitadas. Universitas Psychologica, 10(1), 113-123.

Artículo de investigación.

** Departamento de Psicología Evolutiva y de la Educación, Facultad de Ciencias de la Educación, Universidad de Granada. Campus Universitario La Cartuja s.n. C.P. 18071. Granada (España). Teléfono 958 (34) 249 037; Fax 958 (34) 248 975. E-mails: tpolo@ugr.es; carolina@ugr.es

**** Facultad de Humanidades, Campus Universitario Campus del Carmen, Avenida de las Fuerzas Armadas, 21071, Huelva.E-mail: carmen.diaz@dpsi.uhu. es
RESUMEN

El objetivo de este artículo es conocer las actitudes hacia la discapacidad del alumnado procedente de titulaciones de Ciencias Sociales y Psicología, matriculado en asignaturas en las que se proporciona información sobre la discapacidad. También se pretende determinar la influencia en estas actitudes del contacto con discapacitados. Como instrumento, se aplicó la Escala de Actitudes hacia las Personas con Discapacidad de Verdugo, Jenaro y Arias (1995) a un grupo de 470 estudiantes de la Universidad de Granada. Los resultados mostraron que el alumnado presentaba actitudes positivas hacia los discapacitados, existiendo diferencias según la titulación cursada y siendo además relevante el hecho de mantener contacto con la persona discapacitada. Se discuten los resultados obtenidos y se ofrecen sugerencias para futuras investigaciones.

Palabras clave autores

Actitudes, discapacidad, universidad.

Palabras clave descriptores

Estudiantes con discapacidades, Ciencias Sociales, educación, conducta, mecanismos de conducta.

\section{A B S T R A C T}

The aim of this article is to know the attitudes of university students coming from Social Sciences and Psychology towards people with disabilities. These students are registered in subjects in which information is provided on the disability. It also tries to analyze the influence of the contact with the disabled. The Escala de Actitudes Hacia las Personas con Discapacidad by Verdugo, Jenaro y Arias (1995) was applied. This scale was administered to 470 students from University of Granada. The results showed that the students presented positive attitudes towards the disabled, although there are exist some differences according to the degree studied and being in addition relevant the fact of having maintained contact with the disabled. These findings are discussed in relation to previous research and suggestions for future investigations are considered.

Key words authors

Attitudes, disability, university.

Key words plus

Handicapped students, Social Sciences, education, behavior and behavior mechanisms. 


\section{Introducción}

Las actitudes y expectativas negativas hacia las personas con discapacidad, siguen siendo en la actualidad motivo de análisis, puesto que constituyen una de las principales barreras para la inclusión e integración de las personas con discapacidad. Hoy por hoy, el discapacitado sigue enfrentándose a diversas formas de discriminación relacionadas con prejuicios y actitudes negativas, que se observan tanto en la sociedad en general, como en algunas personas en particular (Jiménez, 2002). Desde la teoría de la acción favorable (Ajzen \& Fishlein, 1980), se entiende por actitud: una evaluación general o un sentimiento global, favorable o no hacia un comportamiento o cuestión. Las actitudes en cuanto son evaluaciones afectivas, muestran significado y juicio, por lo que están abiertas al cambio a través de la reflexión y además son educables (Escámez, García, Pérez \& Llopis, 2007). Modificar las actitudes favorecería una situación de igualdad y la integración social efectiva de las personas con discapacidad (Verdugo \& Arias, 1991). Por este motivo, resulta necesario conocer y evaluar las actitudes hacia la discapacidad, para el desarrollo de programas que hagan posible el cambio de actitudes (Aguado, Alcedo \& Arias, 2008; Aguado, Alcedo \& Flórez, 2003, 2004).

Los estudios realizados para conocer las actitudes hacia la discapacidad han adoptado diferentes perspectivas, en función del colectivo al que se han dirigido (Verdugo, Jenaro \& Arias, 2002). Fundamentalmente, se han analizado las actitudes de madres y familiares, y en el ámbito educativo, las actitudes hacia los discapacitados de maestros y profesionales de la enseñanza (Aguado et al., 2004; Alemany, 2004; Cook, Tankersley, Cook \& Lamdrum, 2000; Rizzo \& Wright, 1988). El objeto de análisis en los diferentes estudios realizados lo han constituido las variables de influencia en el desarrollo de actitudes positivas o negativas, destacándose de acuerdo con García Pastor (1999), que la forma de relacionarnos con las personas con discapacidad podría estar influenciada por nuestras experiencias, y por la forma en que entendemos y definimos la discapacidad.
Se han realizado trabajos que han puesto de manifiesto que la información sobre la discapacidad recibida a lo largo de la formación universitaria, podría ser uno de los determinantes de las actitudes (Carberry, Waxman \& Mckain, 1981; Reina, 2003), comprobándose además que un mayor nivel de información y formación se relaciona con actitudes más positivas (Mestre, Guil, Marcilla, Aguilar \& Gónzalez, 1996; Verdugo et al., 2002). En relación con el hecho de haber mantenido contacto con personas discapacitadas, se han llevado a cabo estudios que pondrían de manifiesto que el contacto tiene una influencia importante tanto en las actitudes negativas como en las positivas; aquellas personas que no han interactuado directamente con personas discapacitadas presentan actitudes de rechazo mayores que aquellas que previamente han tratado con este colectivo. Así, según las investigaciones de Newberry y Parish (1987), el contacto con discapacitados aumentaría las actitudes positivas hacia estas deficiencias. En esta línea encontramos el trabajo de Gómez e Infante (2004), los cuales analizaron las actitudes de los universitarios chilenos hacia las personas con discapacidad, observando que el contacto con la persona discapacitada influye positivamente en las actitudes de los estudiantes. Por otra parte, Yazbeck, McVilly y Parmerter (2004), encontraron que las actitudes de los universitarios hacia las personas con discapacidad intelectual son más positivas que las manifestadas por la población general y éstas, además, estaban relacionadas con el hecho de conocer o mantener un contacto regular con personas discapacitadas. Similares resultados son hallados por Wai y Man (2006), que al analizar las actitudes de profesionales y estudiantes universitarios de psicoterapia, terapia ocupacional, trabajo social y enfermería, hacia personas con discapacidad, observaron que los profesionales mostraban unas actitudes más positivas. Estos resultados parecen estar relacionados con la mayor posibilidad de contacto que estos profesionales mantienen con personas discapacitadas, y con su mayor nivel educativo (Olson \& Zanna, 1993).

En España, diferentes investigaciones han puesto de relieve la influencia del tipo de estudios 
universitarios cursados sobre las actitudes manifestadas (López-Ramos, 2004; Moreno, Rodríguez, Saldaña \& Aguilera, 2006; Polo \& López-Justicia, 2006). Así, López-Ramos (2004) concluye que los alumnos y alumnas procedentes de las carreras de Derecho y Medicina tenían actitudes menos positivas que los de otras titulaciones, incluidas las técnicas, siendo los estudiantes de educación los que presentaban actitudes significativamente más positivas. En esta línea, Moreno et al. (2006) evaluaron las actitudes de alumnos matriculados en materias relacionadas con la atención educativa a las personas con discapacidad, encontrando que, en general, los estudiantes manifestaban una actitud positiva; si bien, existían diferencias significativas en función de si se mantenía o no contacto con personas discapacitadas. Estos resultados, tal y como establece Reina (2003), vendrían a confirmar que las actitudes estarían determinadas por nuestras creencias y estas, a su vez, son producto de nuestras experiencias, conocimientos y de la información disponible.

Polo y López-Justicia (2006), analizaron las actitudes hacia la discapacidad del alumnado de Magisterio, Psicopedagogía, Psicología y Actividad Física de la Universidad de Granada, encontrando que estos estudiantes presentaban actitudes positivas hacia los discapacitados, lo que podría deberse a la influencia de la información y formación recibida en estas titulaciones sobre la temática, así como a la presencia en las titulaciones señaladas, de compañeros con discapacidad. Es evidente que en los últimos años, el porcentaje de alumnos con discapacidad que cursa estudios universitarios se ha incrementado notablemente (De la Red, De la Puente, Gómez \& Carro, 2002); sin embargo, son escasos los estudios que abordan la influencia de este hecho en la percepción y/o actitudes que muestra el alumno sin discapacidad hacia el compañero discapacitado y en torno a la discapacidad en general. En la actualidad, el alumnado con discapacidad en la universidad es más numeroso en titulaciones de Humanidades y Ciencias Sociales, y menos en las Técnicas (Gabinete de Atención Social, 2008), carreras además que en general no cuentan con asignaturas que tratan el tema de la discapacidad, por lo que sus actitudes podrían ser más negativas (Polo \& López-Justicia, 2006). Conocer en qué medida influyen variables como la información sobre la discapacidad y el contacto con estas personas, es crucial para el desarrollo de programas que hagan posible el cambio de actitudes (Aguado et al., 2003, 2004; Aguado et al., 2008), lo que justifica la realización del presente estudio. El propósito de nuestro trabajo es, en primer lugar, conocer las actitudes hacia la discapacidad del alumnado no discapacitado de titulaciones de Ciencias Sociales y Psicología, matriculados en asignaturas que tratan la discapacidad. En segundo lugar, se pretende comprobar si el hecho de mantener contacto con personas discapacitadas influye en las actitudes del alumnado hacia la discapacidad.

\section{Método}

\section{Participantes}

Participaron un total de 470 estudiantes (132 hombres y 338 mujeres) con una media de edad de 19 años que, durante el curso 2007-2008, estaban matriculados en asignaturas en las que en su programa se abordaba la temática de la discapacidad. Dichas titulaciones así como el número de participantes se representan en la Tabla 1.

\section{TABLA 1}

Distribución de alumnos universitarios por centro y titulación

\begin{tabular}{lrrrr}
\hline \multicolumn{1}{c}{ Especialidad } & H & \multicolumn{1}{c}{ M } & \multicolumn{1}{c}{ N } & $\%$ \\
\hline Magisterio & 98 & 212 & 310 & 66,1 \\
Educación Social & 12 & 38 & 50 & 10,6 \\
Psicopedagogía & 10 & 30 & 40 & 8,5 \\
Pedagogía & 7 & 37 & 44 & 9,3 \\
Psicología & 5 & 21 & 26 & 5,5 \\
Total & 132 & 338 & 470 & $100 \%$ \\
\hline
\end{tabular}

Fuente: elaboración propia.

La muestra estaba compuesta en su mayoría por estudiantes de las especialidades de Magisterio 
$(66,1 \%)$ y Educación Social (10,6\%), siendo menor la proporción de alumnos de Psicopedagogía $(8,5 \%)$ y Licenciatura en Psicología (5,5\%).

\section{Instrumento}

El instrumento utilizado para evaluar las actitudes fue la Escala de Actitudes hacia las Personas con Discapacidad (Verdugo et al., 1995). Se trata de una escala multidimensional desarrollada en España, que cuenta con estudios de fiabilidad (alfa de Cronbach 0.92) y validez (una general y otras específicas para deficiencias físicas, sensoriales y mentales). Se considera un instrumento útil, y con las suficientes garantías psicométricas (Verdugo et al., 2002).

La tarea que debe realizar la persona evaluada consiste en opinar si está de acuerdo o no con cada una de las frases que se presentan, formuladas positiva o negativamente, siendo los significados de las opiniones los siguientes: Estoy muy de acuerdo (MA); Estoy bastante de acuerdo (BA); Estoy parcialmente de acuerdo (PA); Estoy parcialmente en desacuerdo (PD); Estoy bastante en desacuerdo (BD); Estoy en total desacuerdo (TD).

El análisis factorial de la Escala puso de manifiesto la existencia de cinco factores. El Factor I, denominado Valoración de Capacidades y Limitaciones (comprende los ítems número 1, 2, 4, 7, 8, $13,16,21,29$, y 36 de la escala), alude a la concepción que el encuestado tiene de las personas con discapacidad. El Factor II, denominado Reconocimiento/Negación de Derechos (aglutina los ítems $6,9,12,14,15,17,22,23,27,35$ y 37$)$ y se refiere al reconocimiento de derechos fundamentales de la persona con discapacidad. El Factor III, Implicación Personal, está formado por juicios referidos a comportamientos concretos de interacción que la persona llevaría a efecto en relación con personas con discapacidad (agrupa los ítems número 3, 5, 10, 11, 25, 26 y 31). El Factor IV (ítems 18, 20, 24, 28 y 34), Calificación Genérica, comprende las atribuciones globales y calificaciones generales que se efectúan acerca de rasgos presuntamente definitorios de la personalidad, o conducta de las personas con discapacidad. El Factor V, Asunción de Roles, consiste en presunciones que el encues- tado realiza sobre la concepción que de sí mismos tienen las personas con discapacidad (engloba los ítems 19, 30, 32 y 33).

Además de los ítems citados en la escala, se recaba información sobre la edad, sexo, estudios y profesión de los participantes. Asimismo, se incluyen ítems relativos al contacto con personas discapacitadas. Se les pregunta si se ha mantenido contacto o no con personas discapacitadas y, en caso afirmativo, la razón del mismo (familiar, laboral, asistencial, ocio/amistad u otras razones), su frecuencia (casipermanente, habitual, frecuente o esporádica) y el tipo de discapacidad que presenta la persona con la que se contacta (física, auditiva, visual, retraso mental o múltiple).

\section{Procedimiento}

La participación de estudiantes universitarios en el estudio se decidió sobre la base de la mayor o menor presencia de estudiantes con discapacidad en los diferentes centros universitarios (Estadística Estudiantes con Discapacidad Curso 2007-2008, Gabinete de Atención Social, Universidad de Granada). Los centros universitarios participantes fueron la Facultad de Psicología y Facultad de Ciencias de la Educación, centros en los que la presencia de universitarios con discapacidad era numerosa, representando el $13 \%$ y $37 \%$, respectivamente.

Una vez elegidos los grupos de los mencionados centros, se contactó al profesorado de las diferentes titulaciones, que impartían asignaturas relacionadas con la educación especial y la discapacidad, y se les solicitó permiso para la administración de la escala en sus clases. Con cada uno de estos docentes, además, se revisaron los programas y contenidos de estas materias con el fin de verificar que el alumnado recibía similar formación tanto en las competencias por adquirir, como en la información proporcionada sobre discapacidad, esto es, definición de discapacidad y diversidad, y características y problemática de los diferentes tipos de discapacidad.

En las diferentes aplicaciones se explicó a los participantes los objetivos de la investigación, de forma que pudieran aportar los comentarios y sugerencias que estimaran convenientes. 


\section{Resultados}

Para la estructuración, ordenación y análisis de los datos se utilizó el paquete de programas estadísticos SPSS en su versión 15 para Windows.

En primer lugar, se realizó un análisis descriptivo de los datos. Para ello partimos de la estructura de la escala en función de cinco factores, en la Tabla 2 se reflejan los resultados obtenidos en cada escala.

\section{TABLA 2}

Estadísticos descriptivos por factores

\begin{tabular}{lcc}
\hline & M & SD \\
\hline Valoración de capacidades y limitaciones & 2,19 &, 63 \\
Reconocimiento/negación de derechos & 1,58 &, 46 \\
Implicación personal & 1,33 &, 48 \\
Calificación genérica & 2,39 &, 68 \\
Asunción de roles & 2,55 &, 84 \\
Media global & 2,01 &, 44 \\
\hline
\end{tabular}

Fuente: elaboración propia.

\section{Valoración de las capacidades y limitaciones}

En líneas generales, se constata el grado de acuerdo entre los estudiantes encuestados respecto a la concepción que tienen de las personas con discapacidad, de su capacidad de aprendizaje y de desempeño, e inferencias sobre aptitudes. Los datos obtenidos indican que, para el alumnado universitario, las personas con discapacidad son tan inteligentes como las personas sin discapacidad $(M=2,16 ; S D=1,15)$. Para la mayor parte de la muestra, las personas con discapacidad tienen una personalidad tan equilibrada como cualquier otra, lo que les permite ser profesionales en igualdad de competencias $(M=2,24 ; S D=1,18)$. Cabe señalar el grado de acuerdo al considerar que en un contexto laboral los discapacitados se entienden sin problemas con el resto de trabajadores $(M=$ 2,28; $S D=1,05)$, en cambio expresaron su desacuerdo ante la afirmación de que las personas dis- capacitadas son poco constantes $(M=2,21 ; S D=$ $1,26)$, admitiendo que puedan ser tan conscientes $(\mathrm{M}=2,12 ; \mathrm{SD}=1,65)$ como las personas que no poseen dicho padecimiento.

\section{Reconocimiento/Negación de derechos}

En este apartado se analizaron las valoraciones en cuanto al reconocimiento de derechos fundamentales de la persona (por ejemplo: igualdad de oportunidades, votar, acceder a créditos, etc.) y, en particular, el derecho que tiene a la normalización y a la integración social. Los universitarios expresaron su total desacuerdo, como reflejan las puntuaciones medias $(M=1,38 ; S D=0,85)$, en cuanto a la opinión de que las personas con discapacidad no deberían vivir con personas afectadas por el mismo problema. Asimismo, se afirmó que los discapacitados deberían tener las mismas posibilidades de acceso al mercado laboral, divertirse con las demás personas, casarse si lo desean, participar en votaciones, etc. $(M=1,85 ; S D=1,04)$.

\section{Implicación personal}

En este bloque se abordan los juicios referidos a comportamientos concretos de interacción que la persona llevaría a efecto en relación con discapacitados. Se constata, como reflejan las medias cercanas a 1, que los estudiantes universitarios encuestados presentan una predisposición favorable a actuar y mostrando una aceptación efectiva de las personas con discapacidad en situaciones sociales, personales y laborales, por ejemplo los encuestados asienten positivamente ante situaciones sociales que faciliten las relaciones sociales $(M=1,16 ; S D$ $=0,66$ ), estando en desacuerdo ante la reclusión social de las personas que padecen discapacidad $(M=1,71 ; S D=1,33)$.

\section{Calificación genérica}

Recoge las atribuciones globales y calificaciones generales que los encuestados efectúan acerca de los rasgos presuntamente definitorios de la personalidad o conducta de las personas con dis- 
capacidad, no apreciándose valoraciones que denoten etiquetajes de tono estereotipado, negativo o peyorativo sobre las personas discapacitadas. Las puntuaciones medias obtenidas a nivel global y diferencial en función de la titulación evidencian un desacuerdo respecto al hecho de que las personas con discapacidad se encuentran siempre de mal humor o resentidas con las personas consideradas "físicamente normales" $(M=1,69 ; S D=1,08)$.

Los universitarios encuestados consideraron que las personas discapacitadas son bastante sociables $(M=2,03 ; S D=1,00)$.

\section{Asunción de roles}

En este apartado se detallan las puntuaciones medias y desviaciones típicas obtenidas en relación con las presunciones que el encuestado efectúa acerca de la concepción que de sí mismos tienen las personas con discapacidad, apreciándose puntuaciones muy similares en las respuestas emitidas. Los encuestados se mostraron bastante de acuerdo, respecto de la consideración de que los que padecen déficit confían, se valoran y se sienten satisfechos consigo mismos $(M=2,63 ; S D=1,24)$. Finalmente, existía un acuerdo entre aquellos estudiantes que cumplimentaron el cuestionario, respecto de que la discapacidad no es un impedimento para llevar una vida normal $(M=1,98 ; S D=1,13)$.
Después de realizar el análisis descriptivo de los diferentes factores que componen la escala, se realizó un análisis multivariado de la varianza (MANOVA), para analizar los efectos que la variable titulación tiene sobre los diferentes factores que componen la escala de actitudes hacia la discapacidad, mostrando igualmente las medias y desviaciones típicas por titulación (Tabla 3).

Encontramos diferencias significativas en cuanto a la titulación de los encuestados en todas los factores (Lambda de Wilks $=2078,0, p<0.000$ ). Analizando estas diferencias observamos (Tabla $3)$, que en consideración a la media global en la escala, $F_{(10,459)}=6,211, p<0.000$, los estudiantes de la diplomatura en Educación Social fueron los que en general, mostraron una actitud más positiva hacia la discapacidad. Además es el grupo con actitudes más favorables en el Factor I (Valoración de capacidades y limitaciones), $F_{(10,459)}=4,854$, $p<0.000$; Factor II (Reconocimiento/negación de derechos), $F_{(10,459)}=4,897, p<0.000$, y en el Factor $\mathrm{V}$ (Asunción de roles), $F_{(10,459)}=2,259, p$ $<0.005$.

En el Factor III (Implicación personal), $F_{(10,459)}$ $=5,205, p<0.000$, los estudiantes de la Licenciatura en Psicopedagogía fue el grupo que puntuó más positivamente. Finalmente en el Factor IV (Calificación genérica), $F_{(10,459)}=4,881, p<$

TABLA 3

Medias y desviaciones típicas según titulación

\begin{tabular}{lccccccc}
\hline \multicolumn{1}{c}{ Factores } & Magisterio & Ed. Social & Psicopedagogía & Pedagogía & Psicología & $F$ & $p$ \\
\hline $\begin{array}{l}\text { Valoración de capacidades } \\
\text { y limitaciones }\end{array}$ & $2,29(0.658)$ & $1,85(0.569)$ & $1,93(0.520)$ & $2,23(0.477)$ & $1,87(0.479)$ & 4,854 & 0.000 \\
$\begin{array}{l}\text { Reconocimiento/negación } \\
\text { de derechos }\end{array}$ & $1,57(0.505)$ & $1,38(0.315)$ & $1,62(0.415)$ & $1,50(0.319)$ & $1,49(0.429)$ & 4,897 & 0.000 \\
Implicación personal & $1,39(0.526)$ & $1,21(0.439)$ & $1,20(0.274)$ & $1,21(0.355)$ & $1,26(0.350)$ & 5,205 & 0.000 \\
Calificación genérica & $2,52(0.698)$ & $2,08(0.613)$ & $2,30(0.594)$ & $2,16(0.621)$ & $1,97(0.465)$ & 4,881 & 0.000 \\
Asunción de roles & $2,66(0.860)$ & $2,20(0.821)$ & $2,53(0.814)$ & $2,39(0.700)$ & $2,32(0.770)$ & 2,559 & 0.005 \\
Media global & $2,10(0.465)$ & $1,74(0.345)$ & $1,91(0.338)$ & $1,90(0.356)$ & $1,78(0.304)$ & 6,211 & 0.000 \\
\hline
\end{tabular}

$* p<0.005$.

Fuente: elaboración propia. 
0.000, los estudiantes de la Licenciatura en Psicología fueron los que puntuaron más positivamente.

A continuación, se hizo un análisis descriptivo para conocer la influencia sobre las actitudes del contacto con personas discapacitadas, mostrando que el 55,5\% de los encuestados declararon no mantener contacto con personas discapacitadas. El $44,4 \%$ restante, expuso haber mantenido contacto con discapacitados principalmente por razones de tipo familiar. En cuanto a la frecuencia del contacto con discapacitados, el 35,7\% comentó que éste era esporádico, mientras para el 54,1\% era frecuente o habitual. La mayor parte de los participantes que habían mantenido contacto con personas discapacitadas lo habían hecho con discapacitados múltiples $(34,9 \%)$ y retrasados mentales $(33,9 \%)$, seguido de discapacitados físicos (22,4\%) auditivos $(7,6 \%)$ y visuales $(1,9 \%)$.

Analizando las diferencias en función del contacto con personas con discapacidad, se encontró que alumnado que había tenido contacto con la persona discapacitada mostró una actitud más positiva hacia las personas con discapacidad que aquellos que no lo habían tenido (Lambda de Wilks $=464,0, p<0.015)$. En la Tabla 4 se muestran las medias y desviaciones típicas en cada una de las subescalas en función del "contacto con personas con discapacidad". También se muestran si existen diferencias significativas entre ambos grupos en cada variable. Los resultados indican que hay diferencias en todos los factores respecto a la variable "contacto con personas discapacitadas".

\section{Discusión}

De acuerdo con los datos obtenidos, la mayoría de los estudiantes universitarios encuestados presentan unas actitudes positivas hacia las personas con discapacidad, siendo relevante, en este sentido, tanto la titulación que realizan como el hecho de haber mantenido contacto con personas discapacitadas.

En cada uno de los factores o variables analizadas en el estudio los alumnos muestran una actitud

TABLA 4

Estadísticos de grupo contacto con discapacidad

\begin{tabular}{lcccccc}
\hline & Contacto & M & SD & ET & F & $p$ \\
\hline \multirow{2}{*}{ Valoración de capacidades y limitaciones } & No & 2,25 & 0.64 & 0.040 & 6,116 & 0.014 \\
& $\mathrm{Si}$ & 2,11 & 0.62 & 0.043 & & \\
Reconocimiento/ negación de derechos & $\mathrm{No}$ & 1,63 & 0.50 & 0.031 & 7,473 & 0.007 \\
& $\mathrm{Si}$ & 1,52 & 0.41 & 0.029 & & \\
Implicación personal & $\mathrm{No}$ & 1,38 & 0.54 & 0.034 & 6,122 & 0.014 \\
& $\mathrm{Si}$ & 1,27 & 0.38 & 0.027 & & \\
Calificación genérica & $\mathrm{No}$ & 2,46 & 0.69 & 0.043 & 5,748 & 0.017 \\
& $\mathrm{Si}$ & 2,31 & 0.67 & 0.047 & & \\
Asunción de roles & $\mathrm{No}$ & 2,65 & 0.84 & .052 & 8,445 & 0.004 \\
& $\mathrm{Si}$ & 2,43 & 0.83 & .058 & & \\
Media global & $\mathrm{No}$ & 2,07 & 0.45 & .028 & 13,60 & 0.000 \\
& $\mathrm{Si}$ & 1,92 & 0.42 & .029 & & \\
\hline
\end{tabular}

$* p<0.005$.

Fuente: elaboración propia. 
favorable hacia la discapacidad. Los universitarios valoran positivamente las capacidades, personalidad y profesionalidad de las personas con discapacidad, defienden su integración y normalización y aceptan de buen grado relacionarse con personas discapacitadas. Similares resultados encontramos en anteriores estudios (Bausela, 2008; Gómez \& Infante, 2004; López-Ramos, 2004; Mella \& González, 2007; Moreno et al., 2006; Santiago \& Escandell, 1998; Upton, Harper \& Wadsworth, 2005; Wai \& Man, 2006; Yazbeck et al., 2004) en los que también se evidencian unas actitudes y percepciones positivas del alumnado universitario hacia la discapacidad.

Sin embargo, no se han hallado valoraciones tan positivas en trabajos que han evaluado las actitudes hacia este colectivo, en otras poblaciones de diferente edad y nivel cultural (Jiménez \& Huete, 2002; Siperstein, Norins, Corbin \& Shriver, 2003; Verdugo et al., 1995). Probablemente, tal y como se ha señalado en otras investigaciones (Chan, Lee, Yuen \& Chan, 2002; Oullette-Kuntz, Burge, Brown \& Arsenault, 2010) un mayor nivel de estudios y formación permitiría tener una visión y un conocimiento más real acerca de la discapacidad, de ahí que las actitudes en universitarios sean más positivas. También ha de tenerse en cuenta que estos trabajos han sido realizados en contextos académicos, sociales y culturales diferentes. Tanto en Estados Unidos, como en algunas universidades europeas, existen, desde hace algunos años, programas de atención y equiparación de oportunidades para estudiantes universitarios; no obstante, el nivel de desarrollo de estos programas no es homogéneo (Alcantud, 2005). Es muy probable que las diferentes acciones llevadas a cabo a nivel político y educativo, aún dirigidas específicamente a la población con discapacidad, supongan también un cambio de actitud hacia la tolerancia y la integración por parte de las personas no discapacitadas en general.

En nuestro trabajo los estudiantes encuestados disponen de información sobre la discapacidad, puesto que estaban matriculados en asignaturas en las que se trata esta temática; no obstante, encontramos diferencias significativas entre las actitudes manifestadas en función de la titulación cursada. Así, observamos que los alumnos de Educación Social mantienen una actitud más positiva hacia la discapacidad, un dato que coincide con los resultados del estudio de López-Ramos (2004), donde los alumnos de Educación Social presentaban unas actitudes más positivas, comparados con sus compañeros de Medicina, Derecho o Trabajo Social. Este hecho podría explicarse debido a que un porcentaje de estudiantes de Educación Social han accedido a la diplomatura, en el cupo de plazas reservadas para los titulados en Ciclos Formativos de Grado Superior de Integración Discapacidad por lo que disponen de información sobre esta temática (López-Justicia, Hernández, Polo, Fernández, Chacón \& González, 2008). Por otra parte, es probable que estos alumnos se encuentren más capacitados para mostrar una actitud de valoración y respeto hacia poblaciones que, como en el caso de los discapacitados, se encuentran en riesgo de exclusión social, ya que en distintas asignaturas de la titulación, aun sin tratarse específicamente de asignaturas que versan sobre discapacidad, el alumno recibe contenidos e información que favorecen estas actitudes positivas. De hecho, estas competencias y actitudes están contempladas en la formación y el ejercicio de la profesión de educador (ANECA, 2004).

En segundo lugar, parece existir relación entre el hecho de tener contacto con personas discapacitadas y mostrar unas actitudes favorables hacia la discapacidad, tal y como anteriores estudios han señalado (Chan et al., 2002; Walsh, Jones, Krause, Obiozor \& Pang, 2008). Cabe destacar, sin embargo, que el hecho de tener compañeros con discapacidad no parece explicar las actitudes positivas encontradas en el alumnado. A pesar de que este estudio se ha llevado a cabo en centros donde la presencia de universitarios con discapacidad era importante, haciendo más probable el contacto entre estudiantes con y sin discapacidad, las razones del contacto en cambio, son de tipo familiar. Investigaciones como las realizadas por Gómez e Infante (2004) y Newberry y Parish (1987), ponen de manifiesto que el contacto directo con el discapacitado es un factor que condiciona las actitudes 
hacia este. En el estudio realizado, las actitudes positivas encontradas estarían relacionadas con un contacto cercano y profundo con la persona con discapacidad, algo característico y habitual en las relaciones familiares. En este sentido, el grado de vinculación y experiencias con la persona que sufre la discapacidad influiría en la percepción y valoración personal hacia la discapacidad.

Por otra parte, sería importante determinar las causas por las que estudiantes con y sin discapacidad parecen no relacionarse. Esta interacción y contacto con compañeros discapacitados podría permitir al alumnado sin discapacidad el descubrimiento de actitudes positivas hacia las personas con discapacidad y, por tanto, posibilitar así una mayor aceptación y entendimiento de las mismas (Verdugo et al., 2002).

Podemos concluir, por tanto, que la titulación cursada y el contacto previo con personas discapacitadas condicionarían unas actitudes positivas hacia la discapacidad. La tolerancia, respeto y aceptación de la persona con discapacidad será mayor en tanto tengamos la oportunidad de conocer y comprender lo que significa la discapacidad. Es un hecho constatado, la presencia cada vez mayor de las personas con discapacidad en las universidades españolas (Polo \& López-Justicia, 2008) y es importante, en este sentido, seguir desarrollando y perfeccionando los programas que en la actualidad tratan de acercar la realidad del discapacitado a la sociedad. Creemos importante continuar promoviendo las campañas de sensibilización y los programas para el cambio de actitudes, pues ambas acciones se han demostrado necesarias para cambiar y mejorar las actitudes hacia la persona discapacitada, incidiendo al mismo tiempo sobre la calidad de vida del discapacitado (Córdoba, Gómez \& Verdugo, 2008). En el ámbito universitario además de demostrarse efectivos (Aguado \& Alcedo, 2007), estos programas serían especialmente convenientes en aquellas titulaciones en las que no existan materias relacionadas con la discapacidad, y por tanto la información sobre esta problemática sea insuficiente o nula.

Por último, es importante comentar ciertas limitaciones del presente estudio. En primer lugar y como consecuencia del instrumento utilizado, no se ha podido obtener información sobre los motivos o razón de la falta de contacto con personas con discapacidad ni tampoco, en caso de que sí lo haya, con quién se ha establecido dicho contacto, cuestiones que, se entiende, podrían ser relevantes en las actitudes del alumnado.

En segundo lugar, sería importante, en futuros trabajos, estudiar las actitudes ante la discapacidad en muestras de universitarios más amplias, confirmando la importancia de las variables contempladas en esta investigación tales como el género, edad, titulación, contacto con personas con discapacidad, y otras no contempladas en el presente trabajo como haber estudiado titulaciones relacionadas con la salud o de carácter más técnico.

\section{Referencias}

Agencia Nacional de Evaluación de la Calidad y Acreditación. (2004). Libro Blanco. Título de Grado en Magisterio (Vol. 1). Madrid. Extraído el 9 de abril, 2009 de http://www.aneca.es/activin/docs/libroblanco jun05 magisterio1.pdf

Aguado, A. L. \& Alcedo, M. A. (2007). Programas de cambio de actitudes hacia personas con discapacidad. Extraído el 2 de agosto, 2008, de http://www.infocop.es/view_article.asp?id $=2157$

Aguado, A. L., Alcedo, M. A. \& Arias, B. (2008). Cambio de actitudes hacia la discapacidad con escolares de primaria. Psicothema, 20(4), 697-704.

Aguado, A. L., Alcedo, M. A. \& Flórez, M. A. (2003). Un programa de cambio de actitudes hacia personas con discapacidad en entorno escolar. Análisis y Modificación de Conducta, 29(127), 673-704.

Aguado, A. L., Alcedo, M. A. \& Flórez, M. A. (2004). Programas de cambio de actitudes ante la discapacidad. Psicothema, 16(4), 667-673.

Ajzen, I. \& Fishbein, M. (1980). Understanding attitudes and predicting social behavior. Englewood Cliff, New Jersey: Prentice Hall.

Alcantud, F. (2005). La integración de los alumnos con n.e.e. en los estudios superiores. En M. López \& R. Carbonell (Coords.), La integración educativa y social. Jornadas Nacionales "Veinte años después de la LISMI" (pp. 137-160). Barcelona: Ariel. 
Alemany, L. Mํㅗ (2004). Las actitudes del profesorado hacia el alumnado con necesidades educativas especiales. Convergencia, 11(34), 183-215.

Bausela, E. (2008). Actitudes hacia la diversidad en un grupo de universitarios de la Universidad Autónoma de San Luís Potosí, México. Revista Científica Electrónica de Psicología, 6, 102-117.

Carberry, H., Waxman, B. \& Mckain, D. (1981). An in-service workshop model for class teachers concerning mainstreaming of the learning disabled child. Journal of Learning Disabilities, 14(1), 26-28.

Chan, C. H., Lee, M. C., Yuen, H. K. \& Chan, F. (2002). Attitudes toward people with disabilities between Chinese rehabilitation and business students: An implication for practice. Rehabilitation Psychology, 47(3), 324-338.

Cook, B. G., Tankersley, M., Cook, L. \& Landrum, T. J. (2000). Teachers' attitudes toward their included students with disabilities. Exceptional Children, 67(1), 115-135.

Córdoba, L., Gómez, J. \& Verdugo, M. A. (2008). Calidad de vida familiar en personas con discapacidad: un análisis comparativo. Universitas Psychologica, 7(2), 369-383.

De la Red, N., De la Puente, R., Gómez, M. C. \& Carro, L. (2002). El acceso a los estudios superiores de las personas con discapacidad física y sensorial. Valladolid: Secretariado de Publicaciones e Intercambio Editorial.

Escámez, J., García, R., Pérez, C. \& Llopis, A. (2007). El aprendizaje de valores y actitudes. Teoría y práctica (Colección Educación en Valores). Madrid: Octaedro/OEI.

Gabinete de Atención Social. (2008). Estadística Estudiantes con Discapacidad Curso 2007-2008 de la Universidad de Granada. Manuscrito no publicado.

García Pastor, C. (1999). Independencia y participación social de personas con necesidades especiales. En G. Pérez Serrano (Coord.), Exclusión e integración social (pp. 149-182). Sevilla: Universidad de Sevilla.

Gómez, V. \& Infante, M. (2004). Actitudes de los estudiantes de educación hacia la integración de las personas con discapacidad y hacia la educación multicultural. Cultura y Educación, 16(4), 371-383.
Jiménez, R. (2002). Las personas con discapacidad en la Educación Superior. Una propuesta para la Diversidad e Igualdad. San José, CR: Fundación Justicia y Género.

Jiménez, A. \& Huete, A. (2002). La discriminación por motivos de discapacidad. Análisis de las respuestas recibidas al cuestionario sobre discriminación por motivos de discapacidad promovido por el CERMI Estatal. Madrid: CERMI.

López-Justicia, M. D., Hernández, C., Polo, M. T., Fernández, C., Chacón, H. \& González, C. (2008). Lectura y escritura en alumnado universitario de Educación de primer curso. Análisis de necesidades. Revista de Educación de la Universidad de Granada, 21 (2), 43-60.

López-Ramos, V. M. (2004). Estudio sobre las actitudes hacia las personas con discapacidad entre los estudiantes de la Universidad de Extremadura. (Tesis doctoral no publicada), Universidad de Extremadura, España.

Mella, S. \& González, L. (2007). Actitudes de los estudiantes de la salud de la Universidad de Chile hacia las personas con discapacidad. Revista Chilena de Terapia Ocupacional, 7, 1-12.

Mestre, J. M., Guil, M. R., Marcilla, A., Aguilar, M. \& González, D. (1996). El nivel de información como determinante de las actitudes hacia la deficiencia mental en familiares de deficientes mentales. Revista de Educación Especial, 22, 17-25.

Moreno, F. J., Rodríguez, I. R., Saldaña, D. \& Aguilera, A. (2006). Actitudes ante la discapacidad en el alumnado universitario matriculado en materias afines. Revista Iberoamericana de Educación, 40, 1-7.

Newberry, M. K. \& Parish, T. S. (1987). Enchancement of attitudes toward handicapped children through social interactions. Journal of Social Psychology, 127(1), 59-62.

Olson, J. M. \& Zanna, M. P. (1993). Attitudes and attitude change. Annual Review of Psychology, 44, 117-154.

Oullette-Kuntz, H., Burge, P., Brown, H. K. \& Arsenault, E. (2010). Public attitudes towards individuals with intellectual disabilities as measured by the concept of social distance. Journal of Applied Research in Intellectual Disabilities, 23(2), 132-142. 
Polo, M. T. \& López-Justicia, M. D. (2006). Actitudes hacia las personas con discapacidad de estudiantes de la Universidad de Granada. Revista Española de Orientación y Psicopedagogía, 17(2), 195-211.

Polo, M. T. \& López-Justicia, M. D. (2008). De la universidad al mundo laboral: estrategias y recursos de acceso al empleo de estudiantes universitarios con discapacidad. [Tesis doctoral]. Granada: Grupo Editorial Universitario.

Reina, R. (2003). Propuesta de intervención para la mejora de actitudes hacia personas con discapacidad a través de actividades deportivas y recreativas. Revista digital. Extraído el 2 de agosto, 2008, de http: www. efdeportes.com

Rizzo, T. L. \& Wright, R. G. (1988). Selected attributes related to physical educators' attitudes toward teaching students with handicaps. Mental Retardation, 26, 307-309.

Santiago, O. \& Escandell, M. O. (1998). Actitudes hacia la discapacidad. Evaluación e intervención psicoeducativa. Revista Interuniversitaria de Psicología de la Educación, 1, 295-314.

Siperstein, G. N., Norins, J., Corbin, S. \& Shriver, T. (2003). Estudio multinacional de actitudes sobre las personas con discapacidad. Boston: Joseph P. Kennedy, Jr. Foundation for the Benefit of Persons with Mental Retardation. Extraído el 20 de abril de 2010, de http://info.specialolympics.org/NR/ rdonlyres/e7bnjytrnebu2zct2o67y2gqs6ifd2e75figxb2opvzjm6qdiusc34u6gyks5np7mskf33dlwwqy- gimvjjunyglbpbd/multinational_study_spanish. pdf

Upton, T. D., Harper, D. C. \& Wadsworth, J. (2005). Postsecondary attitudes toward persons with disabilities: A comparison of college students with and without disabilities. Journal of Applied Rehabilitation Counseling, 36(3), 24-31.

Verdugo, M. A. \& Arias, B. (1991). Evaluación y modificación de las actitudes hacia los minusválidos. Revista de Psicología General y Aplicada, 44(1), 95-102.

Verdugo, M. A., Jenaro, C. \& Arias, B. (2002). Actitudes sociales y profesionales hacia las personas con discapacidad: estrategias de evaluación e intervención. En M. A. Verdugo (Dir.), Personas con discapacidad. Perspectivas psicopedagógicas y rehabilitadoras (pp. 79-135). Madrid: Siglo XXI.

Wai, K. \& Man, D. (2006). Attitudes toward people with disabilities: A comparison between health care professionals and students. International Journal of Rehabilitation Research, 29(2), 155-160.

Walsh, M., Jones, S., Krause, J., Obiozor, E. \& Pang, A. (2008). Attitudes of university students toward individuals with exceptionalities and inclusive practices: A baseline analysis of students enrolled in the introductory course. NERA Conference Proceedings 2008. Paper 22. Extraído el 2 de agosto, 2008, de http://digitalcommons.uconn.edu/nera_2008/2

Yazbeck, M., Mcvilly, K. \& Parmenter, T. (2004). Attitudes toward people with intellectual disabilities. Journal of Disability Policy Studies, 15(2), 97-112. 
